Bull. Austral. Math. Soc.

$46 \mathrm{E} 40,46 \mathrm{~B} 25$

VOL. 59 (1999) [443-447]

\title{
ON COPIES OF THE NULL SEQUENCE BANACH SPACE IN SOME VECTOR MEASURE SPACES
}

\author{
J.C. Ferrando and J.M. Amigó
}

In this note we extend a result of Drewnowski concerning copies of $c_{0}$ in the $\mathrm{Ba}$ nach space of all countably additive vector measures and study some properties of complemented copies of $c_{0}$ in several Banach spaces of vector measures.

\section{Preliminaries}

Throughout this note $(\Omega, \Sigma)$ will be a measure space and $X$ a Banach space. Our notation is standard $[2,3]$. So $b a(\Sigma, X)$ denotes the Banach space over $\mathbb{K}$ of all $X$-valued bounded finitely additive measures defined on $\Sigma$ provided with the semivariation norm

$$
\|F\|(E)=\sup \left\{\sum_{A \in \pi}\left|x^{*} F(A)\right|: x^{*} \in X^{*},\left\|x^{*}\right\| \leqslant 1, \pi \in \mathcal{P}(E)\right\},
$$

for each $E \in \Sigma$, where $\mathcal{P}(E)$ is the class of all finite partitions of $E$ by elements of $\Sigma$. The closed subspace of $b a(\Sigma, X)$ formed by the countably additive measures is denoted by $c a(\Sigma, X)$, while $c c a(\Sigma, X)$ stands for the closed subspace of $c a(\Sigma, X)$ formed by those countably additive measures with relatively compact range. By bvca $(\Sigma, X)$ we denote the Banach space of all $X$-valued countably additive measures $F$ of bounded variation defined on $\Sigma$, equipped with the variation norm $|F|=|F|(\Omega)$. It has been shown in [7] that $(a)$ both $c a(\Sigma, X)$ and bvca $(\Sigma, X)$ contain a copy of $c_{0}$ if and only if either $X$ contains a copy of $c_{0}$ or they contain a copy of $\ell_{\infty}$, and $(b)$ if $\Sigma$ is an infinite $\sigma$-algebra, then $b a(\Sigma, X)$ contains a copy of $c_{0}$ if and only if it contains a copy of $\ell_{\infty}$. If $b a(\Sigma, X)$ contains a complemented copy of $c_{0}$, then $X$ contains a copy of $c_{0}$ [8]. Assuming that each nonzero finite positive measure $\mu \in c a(\Sigma)$ is purely atomic, then $c a(\Sigma, X)$ contains a copy of $c_{0}$ or $\ell_{\infty}$ if and only if $X$ contains respectively a copy of $c_{0}$ or $\ell_{\infty}$ [5, Theorem 2] and, with the same hypotheses, bvca $(\Sigma, X)$ contains a copy of $c_{0}$ or $\ell_{\infty}$ if and only if $X$ contains respectively a copy of $c_{0}$ or $\ell_{\infty}[6]$. On the other hand, $c c a(\Sigma, X)$ contains a copy of $\ell_{\infty}$ if and only if $X$ contains a copy of $\ell_{\infty}[4]$. Note that $c a(\Sigma, X)$ may contain a copy of $c_{0}$ while $X$ does not. Indeed, if $\Omega=[0,1]$ and $\Sigma$ coincides with the $\sigma$-algebra of

Received 29th October, 1998

This paper has been partially supported by DGES grant PB97-0342.

Copyright Clearance Centre, Inc. Serial-fee code: 0004-9729/99 \$A2.00+0.00. 
all Lebesgue measurable subsets of $[0,1]$, then $c a\left(\Sigma, \ell_{2}\right)$ contains a copy of $c_{0},[7]$. In this paper we show by a straightforward technique that, assuming $c c a(\Sigma, X)=c a(\Sigma, X)$, then $c a(\Sigma, X)$ contains a copy of $c_{0}$ if and only if $X$ contains a copy of $c_{0}$. This extends part (B) of Theorem 2 in [5] quoted above. On the other hand, we shall show that if $c a(\Sigma, X)$ or bvca $(\Sigma, X)$ contains a complemented copy of $c_{0}$, then $X$ contains a copy of $c_{0}$. As background of this work we must mention $[4,5,6,7,8]$.

Given a set $A$ of a Banach space $X$, we represent by $[A]$ the closed linear span of $A$. In the sequel we shall shorten to wuC the sentence "weak unconditionally Cauchy". Let us recall that a Banach space is said to have the Schur property if each weakly convergent sequence in $X$ is norm convergent.

\section{Results}

TheOrem 2.1. Assume cca $(\Sigma, X)=c a(\Sigma, X)$. Then ca $(\Sigma, X)$ contains a copy of $c_{0}$ if and only if $X$ contains a copy of $c_{0}$.

ProOF: Let $Z$ denote a copy of $c_{0}$ in $c a(\Sigma, X)=c c a(\Sigma, X)$, and let $J: c_{0} \rightarrow Z$ be a topological isomorphism from $c_{0}$ onto $Z$ such that $F_{n}:=J e_{n}$ for each $n \in \mathbb{N}$ where $\left\{e_{n}: n \in \mathbb{N}\right\}$ is the standard unit vector basis of $c_{0}$. We assume to the contrary that $X$ contains no copy of $c_{0}$. Since $J$ is a bounded linear operator, the series $\sum_{n=1}^{\infty} F_{n}$ is wuC in $c a(\Sigma, X)$ and hence there exists a $C>0$ such that $\sup _{n \in \mathbb{N}}\left\|\sum_{i=1}^{n} \xi_{i} F_{i}\right\|<C\|\xi\|_{\infty}$ for each $\xi \in \ell_{\infty}$. Then, given $E \in \Sigma$, the series $\sum_{n=1}^{\infty} F_{n}(E)$ is wuC in $X$, since for each $x^{*} \in X^{*}$ the map $u: c a(\Sigma, X) \rightarrow \mathbb{K}$ defined by $u(F)=x^{*} F(E)$ is a continuous linear form on $c a(\Sigma, X)$ and, consequently, $\sum_{n=1}^{\infty}\left|x^{*} F_{n}(E)\right|=\sum_{n=1}^{\infty}\left|u F_{n}\right|<\infty$. As we are assuming that $X$ does not contain a copy of $c_{0}$, according to a well known result of Bessaga and Pelczyński $[1]$ the series $\sum_{n=1}^{\infty} F_{n}(E)$ is (BM)-convergent in $X$. So we may consider the linear operator $\varphi: \ell_{\infty} \rightarrow c a(\Sigma, X)$ defined by $\varphi \xi(E)=\sum_{n=1}^{\infty} \xi_{n} F_{n}(E)$. Actually $\varphi \xi \in b a(\Sigma, X)$ and $\varphi$ is a bounded linear operator from $\ell_{\infty}$ into $b a(\Sigma, X)$ since, given $\xi \in \ell_{\infty}$ and $E \in \Sigma$, we have

$$
\|\varphi \xi(E)\| \leqslant \sup _{n \in \mathbb{N}}\left\|\sum_{i=1}^{n} \xi_{i} F_{i}(E)\right\| \leqslant \sup _{n \in \mathbb{N}}\left\|\sum_{i=1}^{n} \xi_{i} F_{i}\right\| \leqslant C\|\xi\|_{\infty} .
$$

So $\|\varphi \xi\| \leqslant 4 C\|\xi\|_{\infty}$, which shows at the same time that $\varphi \xi \in b a(\Sigma, X)$ and that $\varphi$ is a bounded linear operator from $\ell_{\infty}$ into $b a(\Sigma, X)$. Moreover, since $\left\{\sum_{i=1}^{n} \xi_{i} F_{i}\right\}$ is a sequence of $X$-valued countably additive vector measures such that $\lim _{n \rightarrow \infty} \sum_{i=1}^{n} \xi_{i} F_{i}(E)=\varphi \xi(E)$ exists in $X$ for each $E \in \Sigma$, according to the Vitali-Hahn-Saks' theorem [3, Chapter 1, Corollary 4.10] there exists a nonzero finite positive $\mu \in c a(\Sigma)$ such that $\varphi \xi \ll \mu$. 
Consequently $\varphi \xi \in c a(\Sigma, X)$ for each $\xi \in \ell_{\infty}$, which shows that $\varphi\left(\ell_{\infty}\right) \subseteq c a(\Sigma, X)$. As $c a(\Sigma, X)=c c a(\Sigma, X)$ by hypothesis, we have that $\varphi\left(\ell_{\infty}\right) \subseteq c c a(\Sigma, X)$. Then, since

$$
\left\|\varphi e_{n}\right\|=\left\|F_{n}\right\|=\left\|J e_{n}\right\| \geqslant \frac{1}{\left\|J^{-1}\right\|}
$$

for each $n \in \mathbb{N}$, Rosenthal's $\ell_{\infty}$ theorem [10] yields an infinite set $M \subseteq \mathbb{N}$ such that the restriction $\psi$ of $\varphi$ to $\ell_{\infty}(M)$ is a topological isomorphism into $c c a(\Sigma, X)$. According to the above quoted result of [4], this implies that $X$ contains a copy of $\ell_{\infty}$, contradicting the fact that $X$ does not contain a copy of $c_{0}$.

COROLlary 2.2. If $X$ has the Schur property, then $c a(\Sigma, X)$ does not contain a copy of $c_{0}$.

Proof: Since $X$ has the Schur property, then $c c a(\Sigma, X)=c a(\Sigma, X)$. Given that $X$ cannot contain a copy of $c_{0}$, the conclusion follows from Theorem 2.1.

COROLlaRY 2.3. [5, Theorem 2] If every nonzero finite positive measure on $\Sigma$ is purely atomic, then $c a(\Sigma, X)$ contains a copy of $c_{0}$ if and only if $X$ contains a copy of $c_{0}$.

Proof: Suppose that every nonzero finite positive measure on $\Sigma$ is purely atomic and let $G \in c a(\Sigma, X)$ be nonzero. According to a theorem of Bartle-Dunford-Schwartz [3, Chapter 1, Corollary 2.6] there is some $\mu \in c a^{+}(\Sigma)$ such that $G \ll \mu$. By hypothesis $\mu$ is purely atomic, so there exists a finite or infinite pairwise disjoint sequence of atoms $\left\{A_{n}\right\}$ in $\Sigma$ such that $\mu\left(A_{n}\right)>0$ and $\bigcup_{n} A_{n}=\Omega$. Define $g: \Omega \rightarrow X$ such that $g(\omega)=$ $\left(G\left(A_{n}\right) / \mu\left(A_{n}\right)\right)$ whenever $\omega \in A_{n}$ for each $n$. Then, as is well known, $g$ is $\mu$-measurable and $G(E)=(P) \int_{E} g(\omega) d \mu$ [Pettis integral] for each $E \in \Sigma$. But this implies that $G(\Sigma)$ is relatively compact in norm [9, Theorem 10.4.4], and consequently $G \in c c a(\Sigma, X)$. Thus $c a(\Sigma, X)=c c a(\Sigma, X)$ and Theorem 2.1 applies.

THEOREM 2.4. If $c a(\Sigma, X)$ contains a complemented copy of $c_{0}$, then $X$ contains a copy of $c_{0}$.

Proof: Set $Z=\left[F_{n}\right]$, let $J: c_{0} \rightarrow Z$ be a topological isomorphism from $c_{0}$ onto $Z$ where $F_{n}:=J e_{n}$ for each $n \in \mathbb{N}$, and let $P$ be a bounded linear projection from $c a(\Sigma, X)$ onto $Z$. We assume to the contrary that $X$ does not contain a copy of $c_{0}$ and proceed as in the proof of the previous theorem until we show that the linear operator $\varphi: \ell_{\infty} \rightarrow$ $c a(\Sigma, X)$ defined by $\varphi \xi(E)=\sum_{n=1}^{\infty} \xi_{n} F_{n}(E)$ is well-defined and bounded. Consequently, $\varphi\left(\ell_{\infty}\right) \subseteq c a(\Sigma, X)$.

Since $P F_{n}=F_{n}$ for each $n \in M$, the bounded linear operator $Q: \ell_{\infty} \rightarrow c_{0}$ defined by $Q=J^{-1} \circ P \circ \varphi$ satisfies $Q e_{n}=e_{n}$ for each $n \in \mathbb{N}$. In fact,

$$
Q e_{n}=J^{-1} P \varphi e_{n}=J^{-1} P F_{n}=J^{-1} F_{n}=J^{-1} J e_{n}=e_{n},
$$


which implies $Q \zeta=\zeta$ for each $\zeta \in c_{0}$. Hence, if $\xi \in \ell_{\infty}$, as $Q \xi \in c_{0}$ one has

$$
Q^{2} \xi=Q(Q \xi)=Q \xi
$$

But this means that $Q$ must be a bounded projection operator from $\ell_{\infty}$ onto $c_{0}$, a contradiction.

THEOREM 2.5. If bvca $(\Sigma, X)$ contains a complemented copy of $c_{0}$, then $X$ contains a copy of $c_{0}$.

ProOf: Set $Z=\left[F_{n}\right], J: c_{0} \rightarrow Z$ a topological isomorphism from $c_{0}$ onto $Z$ where $F_{n}:=J e_{n}$ for each $n \in \mathbb{N}$, and let $P$ be a bounded linear projection operator from bvca $(\Sigma, X)$ onto $Z$. By assuming to the contrary that $X$ does not contain a copy of $c_{0}$ and reasoning as in the proof of Theorem 2.1 , there is a $C>0$ such that $\sup _{k \in \mathbb{N}}\left|\sum_{j=1}^{k} \xi_{j} F_{j}\right| \leqslant$ $C\|\xi\|_{\infty}$. Define the linear operator $\varphi: \ell_{\infty} \rightarrow b a(\Sigma, X)$ by $\varphi \xi(E)=\sum_{n=1}^{\infty} \xi_{n} F_{n}(E)$. We only need to prove that $\varphi \xi \in b v c a(\Sigma, X)$ and that $\varphi$ is a bounded linear operator. So, if $\left\{E_{i}: 1 \leqslant i \leqslant n\right\}$ is a partition of $\Omega$ by elements of $\Sigma$ then, given $\xi \in \ell_{\infty}$, we have

$$
\begin{aligned}
\sum_{i=1}^{n}\left\|\varphi \xi\left(E_{i}\right)\right\| & =\sum_{i=1}^{n}\left\|\sum_{j=1}^{\infty} \xi_{j} F_{j}\left(E_{i}\right)\right\| \leqslant \sup _{k \in \mathbb{N}} \sum_{i=1}^{n}\left\|\sum_{j=1}^{k} \xi_{j} F_{j}\left(E_{i}\right)\right\| \\
& \leqslant \sup _{k \in \mathbb{N}}\left|\sum_{j=1}^{k} \xi_{j} F_{j}\right| \leqslant C\|\xi\|_{\infty} .
\end{aligned}
$$

So $|\varphi \xi| \leqslant 4 C\|\xi\|_{\infty}$, which shows at the same time that $\varphi \xi \in b v c a(\Sigma, X)$ and that $\varphi$ is a bounded linear operator from $\ell_{\infty}$ into $b v c a(\Sigma, X)$.

AdDEd IN Proof. Since $c a(\Sigma, X) \cong L_{w^{*}}\left(c a(\Sigma)^{*}, X\right)$, Theorems 2.1 and 2.4 can be viewed as particular cases of well known properties of $L_{w^{*}}\left(c a(\Sigma)^{*}, X\right)$.

\section{REFERENCES}

[1] C. Bessaga and A. Pelczyński, 'On bases and unconditional convergence of series in Banach spaces', Studia Math. 17 (1958), 151-164.

[2] J. Diestel, Sequences and series in Banach spaces, Graduate Texts in Math. 92 (Springer-Verlag, New York, Berlin, Heidelberg, Tokyo, 1984).

[3] J. Diestel and J. Uhl, Vector measures, Math Surveys 15 (Amer. Math. Soc. Providence, R.I., 1977).

[4] L. Drewnowski, 'Copies of $\ell_{\infty}$ in an operator space', Math. Proc. Camb. Phil. Soc. 108 (1990), 523-526.

[5] L. Drewnowski, 'When does $c a(\Sigma, Y)$ contain a copy of $\ell_{\infty}$ or $c_{0}$ ?', Proc. Amer. Math. Soc. 109 (1990), 747-752.

[6] J.C. Ferrando, 'When does bvca $(\Sigma, X)$ contain a copy of $\ell_{\infty}$ ?', Math. Scand. 74 (1994), 271-274. 
[7] J.C. Ferrando, 'Copies of $c_{0}$ in certain vector-valued function Banach spaces', Math. Scand. 77 (1995), 148-152.

[8] J.C. Ferrando and J.M. Amigó, 'On copies of $c_{0}$ in the bounded linear operator space', submitted.

[9] J.C. Ferrando, M. López Pellicer and L.M. Sánchez Ruiz, Metrizable barrelled spaces, Pitman Research Notes in Math. 332 (Longman, Essex, 1995).

[10] H.P. Rosenthal, 'On relatively disjoint families of measures, with some applications to Banach space theory', Studia Math. 37 (1979), 13-36.

Depto. Estadística y Matemática Aplicada

Universidad Miguel Hernández

E-03202 Elche (Alicante)

Spain

e-mail: jc.ferrando@umh.es

jm.amigo@umh.es 